\title{
A Comparative study to quantify the area of obturating material, sealer and voids in root canals obturated with Resilon/Epiphany and gutta-percha with different sealers
}

\author{
Vikram M, ${ }^{1 *}$ Shetty $N,{ }^{2}$ Singh $V P^{3}$ \\ ${ }^{1}$ Department of Conservative Dentistry and Endodontics, B.P.Koirala Institute of Health Sciences, Dharan, Nepal, ${ }^{2}$ De- \\ partment of Conservative Dentistry and Endodontics, Manipal College of Dental Sciences, Mangalore, Karnataka, India, \\ ${ }^{3}$ Department of Orthodontics and Dentofacial Orthopaedics, B.P.Koirala Institute of Health Sciences, Dharan, Nepal.
}

*Corresponding Author:

Dr. Mannu Vikram

Assistant Professor,

Department of Conservative Dentistry and Endodontics, B.P.Koirala Institute of Health Sciences,

Dharan, Nepal.

e-mail: drmannuvikram@gmail.com

\section{Citation}

Vikram M, Shetty N, Singh VP. A Comparative study to quantify the area of obturating material, sealer and voids in root canals obturated with Resilon/Epiphany and gutta-percha with different sealers. Nepal Journal of Medical sciences 2014;3(1):8-13.

\begin{abstract}
Background: Complete obturation of the root canal with an inert filling material and creation of a fluid-tight seal are among the major goals of successful endodontic treatment.
\end{abstract}

Methods: Extracted mandibular premolar teeth were decoronated, prepared in a crown-down fashion and irrigated with $2.5 \%$ sodium hypochlorite and $17 \%$ EDTA. The specimens were randomly assigned into 4 groups (n=10/each) and obturated with gutta-percha and Resilon/Epiphany with different sealers. Horizontal sections were obtained of the coronal, middle and apical third at levels of 14-mm, 8-mm and 2-mm from the apex respectively and were viewed under magnification. The area of obturating material, sealer and voids were calculated using Image J software. Statistical comparisons between the Resilon and gutta-percha groups were made with Kruskal-Wallis Test and intergroup comparisions were made by Mann-Whitney $U$ test with Bonferroni correction.

Results: The results of this study showed that root canals obturated with gutta-percha or Resilon with Epiphany as sealer had significantly less area of sealer plus voids and voids alone as compared to root canals obturated with gutta-percha and AH Plus as sealer or gutta-percha and GuttaFlow.

Conclusion: From the results of this study it can be concluded that methacrylate resin-based sealer Epiphany will lead to a better obturation of the root canals and adhesive sealers hold a better prospective.

Keywords : AH Plus; GuttaFlow; Resilon/Epiphany
Background:

The primary objective of endodontic therapy is shaping and cleaning of the root canal system through mechanical and chemical means. The canals are then obturated to obtain a three-dimensional seal of the root canal system, thus eliminating the penetration of oral fluids, microorganisms and periodontal fluid. ${ }^{1}$

The presence of voids in the obturation can be an avenue for microleakage. Leakage through a filled root canal may take place along the sealer-dentine and sealerroot filling material interfaces or through voids within the sealer and the root filling material. ${ }^{2}$ The factors that can 
influence microleakage through the obturating materials are: adaptation of the filling material to the root canal wall, solubility or setting expansion or contraction of the sealer. To achieve success, the root canal filling must seal the canal space both apically and coronally. ${ }^{3,4}$

Among the numerous obturation techniques and filling materials available, gutta-percha continues to be the material of choice, owing to its unique chemical and physical properties. Gutta-percha is an impermeable core material, but there is an absence of a chemical union between guttapercha and the root canal sealer. ${ }^{3}$ Gutta-percha does not bond to internal tooth structure which results in areas of voids, and therefore, the absence of a complete seal. ${ }^{5}$

Although sealers enhance sealing ability, the optimal obturation aims at maximizing the volume of the core material while minimizing the amount of sealer between the inert core and the canal wall. In contrast to gutta-percha, which is chemically and dimensionally stable, the areas filled by sealer are more vulnerable because it can dissolve over time. ${ }^{6}$

The incorporation of restorative dentin-bonding principles may enhance the resistance of obturation materials to leakage. ${ }^{7,8}$ Recently, a thermoplastic, synthetic polymer-based root canal filling material was introduced. The resin core filling material, Resilon (Resilon Research LLC, Madison, CT), is a polyester-based polymer that contains bioactive glass and radiopaque fillers. They attribute the resisitance to leakage and increased sealing properties to the "monoblock" created by the affinity of the Resilon core to the Epiphany resin-based sealer. ${ }^{9}$ Resilon/ Epiphany is the first obturation system to claim the ability to form a "monoblock" between the canal walls and obturation material. $^{7}$

Many different root canal cements are currently being used in combination with gutta-percha to fill the root canal after biomechanical preparation. GuttaFlow (Coltene Whaledent, Alsatten, Switzerland) is another root canal sealer which consists of a mixture of gutta-percha powder, poly-dimethylsiloxane and silver particles. Resin-based sealers like $\mathrm{AH}$ Plus have steadily gained popularity. $\mathrm{AH}$ Plus is an epoxy resin-based sealer, which can form covalent bonds between dentin collagens and resin, preserves its dimensional stability in the long term, and has superior sealing properties. ${ }^{10}$

The purpose of this study is to investigate and compare the cross- sectional area of sealer plus voids together and voids alone in the coronal, middle and apical region of the root canal fillings completed with laterally compacted Resilon and gutta-percha cones with the new resin-based Epiphany sealer and gutta-percha with the conventionally used AH Plus and GuttaFlow.

\section{Methods:}

Forty mandibular human premolars based on similarity in size macroscopically and with straight and single root canals were stored in saline solution at $4{ }^{\circ} \mathrm{C}$ until actual experiments. Their crowns were removed at the cemento-enamel junction by using a water-cooled, lowspeed diamond disc (Mani, Japan). Roots were instrumented with EasyRaCe NiTi rotary files (FKG, Swiss Dental Products) in a crown down technique and enlarged to a size 40/0.04 taper. After each instrument, the canals were irrigated with $5 \mathrm{~mL} 2.5 \%$ sodium hypochlorite $(\mathrm{NaOCl})$ and a final irrigation of $5 \mathrm{~mL} 17 \%$ ethylenediaminetetracetic acid (EDTA) and $5 \mathrm{~mL} 2.5 \% \mathrm{NaOCl}$, after which the canals were flushed with $10 \mathrm{~mL}$ distilled water to avoid the prolonged effect of the irrigating solutions. Root canals were dried with paper points and randomly assigned into 4 obturation groups $(n=10 /$ each $)$.

In group 1, AH Plus sealer was applied into the root canals and a size 40/0.04 taper gutta-percha master point (Dentsply/ Maillefer) was seated into the root canal with tug-back. Lateral compaction was performed and approximately 4 accessory cones were inserted per root canal, and the quality of the filling was confirmed with radiographs. Similarly, in group 2, a master gutta-percha point having a size 40/0.04 taper was selected. A GuttaFlow capsule was selected and mixed for 30 seconds in a triturator. A GuttaFlow dispensing canal tip was attached to the capsule and inserted into the dispenser. The canal tip was inserted into the apical third of the canal $1 \mathrm{~mm}$ short of the working length. A small amount of GuttaFlow was gently dispensed into the apical third. GuttaFlow was directly applied on the master gutta-percha point and then inserted into the canal.

In group 3, RealSeal primer was placed into the canal with a syringe, and after 30 seconds, the excess was removed with a paper point. Thereafter, a size 40, 0.04 taper guttapercha master point (Dentsply/Maillefer) was seated into the root canal with tug-back. Lateral compaction was performed as with group 1 by using accessory gutta-percha cones. In group 4, a size 40, 0.04 taper Resilon cone was inserted to the working length with tug-back. The RealSeal primer was placed into the canal with a syringe, and after 30 seconds, the excess was removed with a paper point. With an insulin syringe, $0.05 \mathrm{~mL}$ of freshly mixed RealSeal sealer 
was injected into the canal. Then, a master Resilon cone (size 40/0.04 taper) was gently seated into the canal and condensed with a \#25 finger spreader. Fine accessory Resilon cones were inserted until they could not be introduced more than $3 \mathrm{~mm}$ into the root canal without measuring the extent of spreader penetration. Approximately 4 accessory cones were inserted per root canal, and the quality of the filling was confirmed with radiographs.

The coronal access of specimen was restored using a hybrid resin composite material (Spectrum TPH; Dentsply), using a total-etch/single-bottle adhesive system (Prime \& Bond NT; Dentsply). All clinical procedures were performed by the same operator.

\section{Sectioning and Image Analysis}

The specimens were stored for 2 weeks at $37^{\circ} \mathrm{C}$ and $100 \%$ humidity to allow the sealer to set completely. Horizontal sections were obtained of the coronal, middle and apical third at levels of 14-mm, 8-mm and 2-mm from the apex respectively. . During sectioning, the specimens were subjected to continuous water cooling to prevent frictional heat and, thus, smearing of Resilon or gutta-percha that could tend to hide areas of sealer. The coronal surfaces of the sections were then digitally photographed at $100 \mathrm{X}$ magnification under a light microscope and transferred to an IBM-compatible PC and saved as Adobe (Adobe, San Jose, CA) files. Using Image J (Wayne Rasband; National Institute of Health, Bethesda, MA) software, the crosssectional area of the root canal (RC area) and the area filled by the sealer (and voids, if present) was calculated.

For each specimen, the ratio of sealer plus voids to root canal area was calculated by dividing sealer plus void area to the root canal area. The ratio of voids (alone) was also calculated by dividing the area of voids by the root canal area. Thereafter, statistical analysis of the data was done. For each section (coronal, middle and apical) statistical comparisons between the Resilon and gutta-percha groups were made with Kruskal-Wallis Test and intergroup comparisions were made by Mann-Whitney $U$ test with Bonferroni correction. Differences within each obturation group for each level of sectioning was analyzed statistically.

\section{Results:}

The results of this study evaluated the ratios of the crosssectional areas of sealer plus voids and the cross-sectional area of voids to that of the root canal presented in table number as mean and standard deviation.

Mann Whitney U Test with Bonferroni correction was used where $\mathrm{p}$ value $=0.0125$ is considered as statistically significant. Group 4 (Resilon+Epiphany) showed better results comared to all other experimental groups with respect to sealer + void or voids at all three levels tested (Table 1).

\begin{tabular}{|c|c|c|c|c|}
\hline 牙 & & $\begin{array}{ll}1 & \\
+ & \\
\infty & 0 \\
0 & 0 \\
0 & 0 \\
0\end{array}$ & $\begin{array}{l}\dot{1} \\
\overline{0} \\
\overline{0} \\
0 \\
0\end{array}$ & $\begin{array}{l}1 \\
+ \\
+ \\
8 \\
8 \\
0 \\
0 \\
0\end{array}$ \\
\hline $\mathscr{\simeq}$ & & 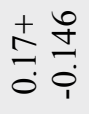 & $\begin{array}{l}\stackrel{+}{\sim} \hat{0} \\
\stackrel{0}{0}\end{array}$ & 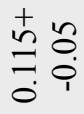 \\
\hline 贡 & & 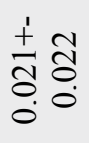 & $\begin{array}{l}1 \\
t \\
8 \\
8 \\
0 \\
0\end{array}$ & $\begin{array}{l}\stackrel{1}{+} 0 \\
\stackrel{0}{0} \\
0 \\
0 \\
0\end{array}$ \\
\hline ثิ & & $\frac{1}{4} \frac{0}{0}$ & $\frac{1}{+} \frac{1}{0}$ & $\begin{array}{l}+1 \\
\stackrel{1}{0} \\
\dot{0} \\
\dot{0}\end{array}$ \\
\hline 峲 & & 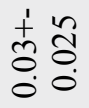 & $\stackrel{1}{ \pm} \underset{0}{\stackrel{1}{0}}$ & $\begin{array}{l}\frac{1}{t} \\
0 \\
0 \\
0\end{array}$ \\
\hline ঠ & & $\begin{array}{l}+1 \\
\stackrel{+}{n} \\
\\
0\end{array}$ & 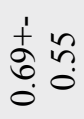 & $\begin{array}{l}\dot{1} \\
\stackrel{n}{n} \\
\dot{b} \\
\dot{0}\end{array}$ \\
\hline$\frac{ \pm}{ \pm}$ & 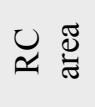 & $\begin{array}{ll}+ & 0 \\
m & + \\
0 & + \\
0 & 0\end{array}$ & $\begin{array}{l}+ \\
+ \\
\stackrel{+}{+}\end{array}$ & $\begin{array}{l}1 \\
+ \\
+ \\
\end{array}$ \\
\hline Ө & 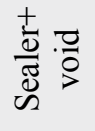 & $\begin{array}{l}+1 \\
\underset{n}{2} \\
0 \\
0\end{array}$ & $\frac{ \pm}{n}$ & 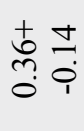 \\
\hline & $=$ & 으 & 으 & 으 \\
\hline & 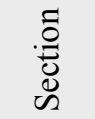 & 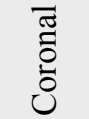 & $\frac{0}{\overline{0}}$ & $\frac{\pi}{\frac{0}{2}}$ \\
\hline
\end{tabular}

Upon comparisons within the three levels of obturation groups, Group 4 (Resilon+Epiphany) showed statistically significant results at coronal and middle levels with respect to cross-sectional area of sealer + voids as well as voids to the root canal area (Table 2, 3, 4 and 5).

Table 2: Coronal section- sealer+void/rc area

\begin{tabular}{ccc}
\hline Group & $\mathbf{Z}$ & p value \\
\hline GPAH+ / GPEP & -3.402 & 0.001 \\
GPGF / GPEP & -3.704 & 0.000 \\
GPGF / ReEP & -3.477 & 0.001 \\
\hline
\end{tabular}

Table 3: Coronal section - void/rc area

\begin{tabular}{lcc}
\multicolumn{1}{c}{ Group } & \multicolumn{1}{c}{$\mathbf{Z}$} & p value \\
\hline GPAH+ / ReEP & -2.92 & 0.003 \\
GPGF / ReEP & -3.56 & 0.000
\end{tabular}


GPEP / ReEP

$-2.84$

0.004

Table 4: Middle section - sealer+void / RC area

\begin{tabular}{lcc}
\hline Group & $\mathrm{Z}$ & $\mathrm{p}$ value \\
\hline GPAH+ / GPEP & -2.571 & 0.010 \\
GPAH+ / ReEP & -2.87 & 0.004 \\
GPGF / GPEP & -3.100 & 0.002 \\
GPGF / ReEP & -3.25 & 0.001 \\
\hline
\end{tabular}

Table 5: Middle section - void / RC area

\begin{tabular}{ccc}
\hline Group & $\mathrm{Z}$ & $\mathrm{p}$ value \\
\hline $\mathrm{GPAH}+$ / GPEP & -3.59 & 0.000 \\
$\mathrm{GPAH}+/$ ReEP & -3.17 & 0.001 \\
$\mathrm{GPGF} / \mathrm{GPEP}$ & -3.96 & 0.000 \\
$\mathrm{GPGF} / \mathrm{ReEP}$ & -3.65 & 0.000 \\
\hline
\end{tabular}

At the apical level, all the experimental groups were similar with no statistically significant difference (Table 6).

Table 6: Apical section - sealer+void / RC area

\begin{tabular}{ccc} 
Group & $\mathrm{Z}$ & $\mathrm{p}$ value \\
\hline $\mathrm{GPAH}+/ \mathrm{ReEP}$ & -2.988 & 0.003 \\
$\mathrm{GPGF} / \mathrm{ReEP}$ & -2.648 & 0.008
\end{tabular}

Apical section -void / RC area ( not significant )

$\mathrm{P}<0.0125$ is considered as statistically significant.

\section{Discussion:}

Coronal leakage into the obturated root canal system had not received significant attention until the late 1980s. Since then, there have been numerous dye, bacterial penetration, and fluid filtration leakage studies that have evaluated coronal leakage. ${ }^{11}$ Contemporary adhesive strategies used for intracoronal adhesive seals attempt to eliminate leakage and strengthen tooth structures by creating monoblocks between tooth substrates and restorative materials, with increasing success and predictability. Likewise, potential improvements in apical and coronal seals and strengthening of endodontically treated teeth may be anticipated by establishing similar monoblocks between the intraradicuar dentin and adhesive root fillings. ${ }^{12}$

Resilon (Resilon Research LLC, Madison, CT) as an alternative root filling material offers the promise of adhesion to root dentine. Resilon, a thermoplastic filled polymer, contains methacryloxy groups and thus can be used in conjunction with a resin-base sealer such as Epiphany. ${ }^{13}$ When Resilon is applied using a methacrylatebased sealer, like Epiphany to self-etching primer-treated root dentin, it creates a secondary monoblock. ${ }^{14}$ Obturation with this system suggests creating a bonded seal with the dentinal tubules within the root canal system. Thus, the core material, sealer and dentinal tubules become a single solid structure. ${ }^{10}$

AH Plus sealer is considered as the gold standard against which all new sealers and root canal obturation materials are compared. Although $\mathrm{AH}$ Plus has adequate longterm dimensional stability, its sealing ability remains controversial partly because AH Plus does not bond to guttapercha. ${ }^{15}$ In 2004, Coltene Whaledent Inc (Cuyahoga Falls, $\mathrm{OH}$ ) introduced a cold, flowable, self-curing obturation material for root canals that combines gutta-percha and sealer into one injectable system. GuttaFlow is available in capsules and can be injected directly into the canal. It is used in combination with a master gutta-percha cone and does not require any form of manual compaction for placement.

The quality of the root canal filling is important not only in the apical third but along the entire length of the root canal. Assuming that minimal sealer thickness and fewer voids are good measures of long term sealing ability, this study was designed to quantify and compare the presence of sealer and voids at the apical, middle and coronal thirds of the root canals surmising that these levels of the root canals would be representative of the entire canal system as it would be difficult to study the canal system as one single unit.

There was a significant statistical difference between guttapercha/AH Plus and gutta-percha/Epiphany for both the variables of sealer+void/root canal area as well as for void/ root canal area. This may be attributable to the fact that Epiphany forms a chemical bond to the root canal dentinal walls unlike the epoxy resin-based sealer AH Plus. This has been shown in a study by Ungor et al where the authors found the highest bond strength between gutta-percha/ Epiphany combination. ${ }^{16}$

There was significant difference between gutta-percha/AH Plus and Resilon/Epiphany. The manufacturers of Resilon/ Epiphany claim that this system allows the formation of 'monoblock' where the Epiphany sealer adheres to the Resilon core material as well as the root canal dentinal wall, thus forming one single unit. This allows for a comparatively lesser void formation and a better seal against microeakage. ${ }^{17}$ These findings are in agreement with a study done by Bouillaguet et al (2007) where the authors used an automatic flow-recording device (Flodec System) to measure microleakage and found that the root canals filled with gutta-percha/AH Plus leaked significantly more than 
those obturated with Resilon/Epiphany. ${ }^{4}$ This is in contrast to a study done by Belli et al in which the authors did a microleakage study using a fluid transport model and found that the quality of apical seal achieved with Resilon core material and Epiphany (RealSeal) sealer is not superior to gutta-percha and AH Plus sealer. ${ }^{14}$

There was a significant statistical difference between guttapercha/GuttaFlow and Resilon/Epiphany. The relatively good performance of Resilon/Epiphany is congruent with other reports (Stratton et al. 2006, Tunga \& Bodrumlu 2006). The formation of a hybrid layer takes place in case of adhesive sealers like Epiphany which is absent in the case of GuttaFlow. This hybrid layer is formed by the achievement of the bond between Epiphany sealer and root canal dentin and when the monomers penetrate into the conditioned dentin surface to create a micromechanical interlocking between the dentin collagen and resin. ${ }^{18}$

The results of our study found that there was no significant difference between root canals obturated with Resilon/ Epiphany (group 4) and gutta-percha/Epiphany (group 3) in either of the two variables. The findings of our study was in accordance with the study done by Gulsahi et al where the authors found no significant differences between the two groups. ${ }^{19}$ Although gutta-percha and Epiphany combination does not have the definite advantage of the chemical bond that exists between Resilon and Epiphany, yet it is believed that gutta-percha is more compactable than Resilon which may account for the otherwise expected result. ${ }^{16}$ The lateral compaction method of obturation done in the present study further lends support to this explanation. The findings in our study is in contrast to the study done by Shipper et al wherein the authors have reported significantly higher leakage with the gutta-percha and Epiphany combination, compared with that of the Resilon plus Epiphany system.

At the coronal level a significant difference was found when gutta-percha/GuttaFlow was compared to guttapercha/Epiphany and Resilon/Epiphany. In case of Resilon/ Epiphany, the sealer bonds to both the core material as well as the dentinal wall, whereas in case of gutta-percha/ Epiphany, the sealer has a chemical union with the dentinal wall but with the core material, no such bonding takes place. This could lead to gaps between the gutta-percha and Epiphany interface.

Resilon/Epiphany had better performance at the middle level than gutta-percha/AH Plus . In case of Resilon/ Epiphany, there is a micromechanical retention via the formation of a thin hybrid layer to the self-etching primer- treated root dentin and chemical coupling of the urethane dimethacrylate-containing Resilon root-filling material to the methacrylate-based sealer and therefore a continuum is anticipated leading to lesser void formation. ${ }^{20}$

At the apical level, there was no significant statistical difference between gutta-percha/Epiphany and Resilon/ Epiphany in terms of sealer+void/root canal area $(p=0.059$ and $\mathrm{z}=-1.891$ ). This is in agreement with a previous study by Gulsahi et al where the authors reported a similar result. ${ }^{25}$ Since, gutta-percha is more compactable than Resilon, this might partially compensate for the lack of monoblock effect as found in the Resilon/Epiphany system. Root canals have high cavity configuration factor ( $\mathrm{C}$-factor) that contribute to polymerization stresses created by resin-based materials along the root canal walls. There was no statistical significant difference between any of the groups in terms of void/ root canal area at the apical level. In the present study, the volume of sealer was minimized at the apical third because the root canal preparation was calibrated to the specific taper of gutta-percha and Resilon cones. Since, we have used NiTi instruments with definite taper to prepare the root canals, a standardization was maintained and we obtained a similar tug-back while obturation in all the specimens used in this study. The apical cross-section was at $2 \mathrm{~mm}$ from the apex. At this level, most of the root canal space was occupied by the master gutta-percha or Resilon cone, with very little or no void at all. This could provide an explanation for the lack of significant difference between the groups.

\section{Conclusion}

Although adhesive products hold promise for future, a number of technical hurdles need to be overcome to maximize the potential benefits of adhesive root canal fillings. Further studies should be performed in evaluating the quality of apical as well as coronal seal, if adhesive concepts are to be incorporated for a better endodontic therapy.

\section{References}

1. Silveira FF, Soares JA, Nunes E, et al. Negative influence of continuous wave technique on apical sealing of the root canal system with Resilon. J Oral Sci 2007;49:121-8.

http://dx.doi.org/10.2334/josnusd.49.121

2. Monticelli F, Sword J, Martin RL, et al. Sealing properties of two contemporary single-cone obturation systems. Int Endod J 2007;40:374-85. http://dx.doi.org/10.1111/j.1365-2591.2007.01231.x

3. Raina R, Loushine RJ, Weller RN, et al. Evaluation of 
the quality of the apical seal in Resilon/Epiphany and Gutta-Percha/AH Plus-filled root canals by using a fluid filtration approach. J Endod 2007;33:944-7. http://dx.doi.org/10.1016/j.joen.2007.05.003

4. Bouillaguet S, Shaw L, Barthelemy J, et al. Longterm sealing ability of Pulp Canal Sealer, AH-Plus, GuttaFlow and Epiphany. Int Endod J 2008;41:219-26. http://dx.doi.org/10.1111/j.1365-2591.2007.01343.x

5. Ingle JI, Bakland LK. Endodontics 5th ed, BC Decker, 2002 .

6. De-Deus G, Coutinho-Filho $\mathrm{T}$, Reis $\mathrm{C}$, et al. Polymicrobial leakage of four root canal sealers at two different thicknesses. J Endod 2006;32:998-1001. http://dx.doi.org/10.1016/j.joen.2006.04.003

7. Fransen JN, He J, Glickman GN, et al. Comparative assessment of ActiV GP/glass ionomer sealer, Resilon/ Epiphany, and gutta-percha/AH plus obturation: a bacterial leakage study. J Endod 2008;34:725-7. http://dx.doi.org/10.1016/j.joen.2008.03.006

8. Pawińska M, Kierklo A, Marczuk-Kolada G. New technology in endodontics--the Resilon-Epiphany system for obturation of root canals. Adv Med Sci. 2006;51 Suppl 1:154-7.

9. Wedding JR, Brown CE, Legan JJ, et al. An in vitro comparison of microleakage between Resilon and gutta-percha with a fluid filtration model. J Endod 2007;33:1447-9.

http://dx.doi.org/10.1016/j.joen.2007.08.021

10. Kaya BU, Kececi AD, Belli S. Evaluation of the sealing ability of gutta-percha and thermoplastic synthetic polymer-based systems along the root canals through the glucose penetration model. Oral Surg Oral Med Oral Pathol Oral Radiol Endod 2007;104:e66-73. http://dx.doi.org/10.1016/j.tripleo.2007.06.024

11. Biggs SG, Knowles KI, Ibarrola JL, et al. An in vitro assessment of the sealing ability of resilon/epiphany using fluid filtration. J Endod 2006;32:759-61. http://dx.doi.org/10.1016/j.joen.2005.08.013
12. Tay FR, Hiraishi N, Pashley DH, et al. Bondability of Resilon to a methacrylate-based root canal sealer. J Endod 2006;32:133-7. http://dx.doi.org/10.1016/j.joen.2005.10.026

13. Baumgartner G, Zehnder M, Paqué F. Enterococcus faecalis type strain leakage through root canals filled with Gutta-Percha/AH plus or Resilon/Epiphany. J Endod 2007;33:45-7. http://dx.doi.org/10.1016/j.joen.2006.08.002

14. Belli S, Ozcan E, Derinbay O, et al. A comparative evaluation of sealing ability of a new, self-etching, dual-curable sealer: hybrid root SEAL (MetaSEAL). Oral Surg Oral Med Oral Pathol Oral Radiol Endod 2008;106:e45-52.

http://dx.doi.org/10.1016/j.tripleo.2008.07.027

15. Bouillaguet S, Shaw L, Barthelemy J, et al. Longterm sealing ability of Pulp Canal Sealer, AH-Plus, GuttaFlow and Epiphany. Int Endod J 2008;41:219-26. http://dx.doi.org/10.1111/j.1365-2591.2007.01343.x

16. Ungor M, Onay EO, Orucoglu H. Push-out bond strengths: the Epiphany-Resilon endodontic obturation system compared with different pairings of Epiphany, Resilon, AH Plus and gutta-percha. Int Endod J 2006;39:643-7.

http://dx.doi.org/10.1111/j.1365-2591.2006.01132.x

17. Pawińska M, Kierklo A, Marczuk-Kolada G. New technology in endodontics--the Resilon-Epiphany system for obturation of root canals. Adv Med Sci 2006;51 Suppl 1:154-7.

18. Gogos C, Economides N, Stavrianos C, et al. Adhesion of a new methacrylate resin-based sealer to human dentin. J Endod 2004;30:238-40. http://dx.doi.org/10.1097/00004770-200404000-00014

19. Gulsahi K, Cehreli ZC, Onay EO, et al. Comparison of the area of resin-based sealer and voids in roots obturated with Resilon and gutta-percha. J Endod 2007;33:1338-41.

http://dx.doi.org/10.1016/j.joen.2007.06.015

20. Weine FS. Endodontic Therapy 6th Ed. Elsevier, 2003. 